\title{
Khảo sát thứ tự thụ đắc các hình thức "li”" từ li họ̣p tiếng Trung Quốc của sinh viên Việt Nam
}

\section{A study on the acquisition order of the separated forms of Chinese separate words by Vietnamese students}

\author{
Lưu Hớn V $\tilde{u}^{1 *}$ \\ ${ }^{1}$ Trường Đại học Ngân hàng Thành phố Hồ Chí Minh, Việt Nam \\ *Tác giả liên hệ, Email: luuhonvu@ gmail.com
}

THÔNG TIN

DOI: $10.46223 / \mathrm{HCMCOUJS}$. soci.vi.16.2.1890.2021

Ngày nhận: 23/05/2021

Ngày nhận lại: 18/08/2021

Duyệt đăng: 18/08/2021

Tù khóa:

hình thức "li"; sinh viên Việt Nam; thứ tự thụ đắc; tiếng Trung Quốc; từ li hợp

Keywords:

separated forms; Vietnamese students; order of acquisition; Chinese; separable words

\section{TÓM TÁtT}

Trên cơ sở Kho ngũ liệu ngôn ngũ trung gian tiếng Trung Quốc của sinh viên Việt Nam, với hai tiêu chí thứ tự tần suất sử dụng chính xác và thứ tự theo ma trận Guttman, bài viết đã xây dựng thứ tự thụ đắc các hình thức "li” từ li hợp tiếng Trung Quốc của sinh viên Việt Nam. Kết quả như sau: hình vị "động" + định ngữ mang tính vị từ + hình vị "tân" > hình vị "động" + hình vị "động" + hình vị "tân" > hình vị "động" + bổ ngữ chỉ khả năng + hình vị "tân" > hình vị "động" + bổ ngữ chỉ số lượng + hình vị "tân" > hình vị "động" + trợ từ động thái 了/ 着/过 + hình vị “tân" > hình vị "động" + bổ ngữ chỉ kết quả + hình vị "tân" > hình vị "động" + định ngữ mang tính thể từ + hình vị "tân" > hình vị "động" + bổ ngữ chỉ hướng + hình vị "tân" > hình vị "tân" + hình vị "động" > hình vị "động" + bổ ngữ chỉ mức độ + hình vị "tân" > hình vị "động" + trợ từ 的 + hình vị "tân" > hình vị “động" + bổ ngữ chỉ tình thái + hình vị "tân". Kết quả nghiên cứu này có giá trị tham khảo nhất định trong việc biên soạn giáo trình và đề thi tiếng Trung Quốc cho sinh viên Việt Nam.

\section{ABSTRACT}

Based on the Chinese interlanguage corpus of Vietnamese students, with the correct use frequency order and the order of the Guttman scale as the standard, this article has constructed the acquisition order of the separated forms of Chinese separable words for Vietnamese students. The result is as follows: verb morpheme + predicate attributive + object morpheme $>$ verb morpheme + verb morpheme + object morpheme $>$ verb morpheme + possible complement + object morpheme $>$ verb morpheme + quantity complement + object morpheme $>$ verb morpheme + dynamic auxiliary verb + object morpheme $>$ verb morpheme + result complement + object morpheme > verb morpheme + subjective attributive + object morpheme $>$ verb 
morpheme + directional complement + object morpheme $>$ object morpheme + verb morpheme $>$ verb morpheme + degree complement + object morpheme > verb morpheme + auxiliary word “的” + object morpheme > verb morpheme + modal complement + object morpheme. The results of this study have certain reference value in compiling Chinese textbooks and exam questions for Vietnamese students.

\section{Giới thiệu}

Từ li hợp (separable words) là loại từ ngữ vừa có thể sử dụng ở hình thức "hợp", vừa có thể sử dụng ở hình thức "li." Đây là loại từ ngữ vô cùng đặc biệt của tiếng Trung Quốc, không có trong các ngôn ngữ khác. Chính vì vậy, từ li hợp đã trở thành một điểm khó trong quá trình học tập và thụ đắc tiếng Trung Quốc của người học nói chung, sinh viên Việt Nam nói riêng.

Các nghiên cứu về thụ đắc từ li hợp hiện nay chủ yếu xoay quanh phân tích lỗi sử dụng của sinh viên Việt Nam, như các nghiên cứu của Luu (2021), H. T. T. Nguyen (2017), H. L. U. Nguyen (2019), Truong (2017), Vuong (2008), ... Nghiên cứu đề cập đến thứ tự thụ đắc các hình thức "li" từ li hợp của sinh viên Việt Nam hiện nay vẫn chưa nhiều. Trong số các tài liệu chúng tôi thu thập được, chỉ có luận văn thạc sĩ của Ngo (2007) đề cập đến vấn đề này.

Trong nghiên cứu của mình, Ngo (2007) đã sử dụng phiếu điều tra để thu thập ngữ liệu của người học, thứ tự thụ đắc được xây dựng trên cơ sở trị trung bình của số thứ tự qua ba bước sau: Bước 1 , sắp xếp thứ tự của các hình thức "li" ở mỗi giai đoạn sơ cấp, trung cấp, cao cấp dựa vào tỉ lệ chính xác từ cao xuống thấp của các hình thức "li" ở mỗi giai đoạn này, kết quả có được ba dãy thứ tự cho ba giai đoạn sơ cấp, trung cấp, cao cấp; Bước 2 , tiến hành tính trị trung bình các số thứ tự của các hình thức "li" dựa trên kết quả của Bước 1 ; Bước 3 , xác định thứ tự thụ đắc của các hình thức "li" bằng cách dựa vào thứ tự từ thấp lên cao của trị trung bình có được ở Bước 2. Có thể thấy, việc tính toán và dựa vào trị trung bình số thứ tự để sắp xếp thứ tự thụ đắc như thế này đã vi phạm nguyên lí thống kê: "các số thứ tự không được phép thực hiện các phép toán cộng trừ nhân chia" (Zhang \& Xu, 2009, pp. 16-17). Vì vậy, thứ tự thụ đắc mà nghiên cứu của Ngo (2007) có được có độ tin cậy không cao.

Từ thực trạng trên, chúng tôi nhận thấy việc tiến hành nghiên cứu lại thứ tự thụ đắc các hình thức "li" từ li hợp tiếng Trung Quốc của sinh viên Việt Nam là hết sức cần thiết. Trong phạm vi bài viết này, chúng tôi sẽ tiến hành khảo sát thứ tự thụ đắc dựa trên cơ sở Kho ngũ liệu ngôn ngũ trung gian tiếng Trung Quốc của sinh viên Việt Nam (phiên bản 2018) do chúng tôi tự xây dựng (quy mô khoảng 906,000 chữ).

\section{Cơ sở lí luận}

Cơ sở lí luận mà chúng tôi sử dụng trong bài viết này là Giả thuyết về thứ tự thụ đắc tự nhiên của Krashen (1985). Krashen cho rằng, chúng ta có thể dự đoán một thứ tự thụ đắc tự nhiên của người học ngôn ngữ, có những điểm ngôn ngữ người học sẽ thụ đắc trước, có những điểm ngôn ngữ người học sẽ thụ đắc sau. Thứ tự thụ đắc tự nhiên không giống với thứ tự về độ khó của những điểm ngôn ngữ, cũng không giống với thứ tự những điểm ngôn ngữ được giảng dạy trên lớp. Việc giảng dạy ngôn ngữ trên lớp không thể thay đổi thứ tự thụ đắc tự nhiên của người học.

\section{Phương pháp nghiên cứu}

Theo Luu (2016), hiện nay có nhiều phương pháp xây dựng thứ tự thụ đắc, như: căn cứ vào thứ tự từ cao xuống thấp của tỉ lệ chính xác (Yang, 2003); tổng hợp thứ tự tần suất sử dụng 
của người bản ngữ, thứ tự tần suất sử dụng của người học và thứ tự tỉ lệ chính xác trung bình của người học (Xiao \& Zhou, 2009), tổng hợp thứ tự tî̉ lệ chính xác trung bình và thứ tự theo ma trận Guttman (Shi, 2012), tổng hợp thứ tự tần suất sử dụng của người bản ngữ, thứ tự tần suất sử dụng chính xác của người học, thứ tự tỉ lệ chính xác của người học và thứ tự theo ma trận Guttman (Huang \& Xiao, 2012).

Tuy nhiên, không tồn tại mối liên hệ giữa thứ tự thụ đắc của người học và tần suất sử dụng của người bản ngữ, vì vậy không cần quan tâm đến thứ tự tần suất sử dụng của người bản ngữ khi xây dựng thứ tự thụ đắc của người học. Tần suất sử dụng và tần suất sử dụng chính xác đều là số lần sử dụng, khác biệt ở chỗ tần suất sử dụng không phân biệt việc sử dụng của người học có chính xác hay không, còn tần suất sử dụng chính xác chỉ quan tâm đến việc sử dụng chính xác của người học, nhưng thụ đắc quan tâm nhiều hơn đến việc sử dụng chính xác. Tỉ lệ chính xác trung bình, tỉ lệ chính xác và ma trận Guttman đều là tỉ lệ chính xác, vì vậy chỉ cần quan tâm đến một trong ba tiêu chí này là được (Luu, 2020).

Luu $(2016,2020)$ còn cho rằng, tỉ lệ chính xác không phản ánh được tình trạng sinh viên tránh sử dụng những điểm ngôn ngữ mà sinh viên biết mình dễ sai khi sử dụng, còn thứ tự tần suất sử dụng chính xác không phản ánh được tỉ lệ chính xác của tần suất sử dụng. Nếu chỉ dựa vào một trong hai tiêu chí này để xây dựng thứ tự thụ đắc có thể sẽ ảnh hưởng đến mức độ chính xác của thứ tự thụ đắc. Vì vậy, trong nghiên cứu này chúng tôi căn cứ vào cả hai tiêu chí thứ tự tần suất sử dụng chính xác và thứ tự theo ma trận Guttman để xây dựng thứ tự thụ đắc các hình thức "li" từ li hợp tiếng Trung Quốc của sinh viên Việt Nam.

\section{Kết quả nghiên cứu}

\section{Việt Nam}

\subsection{Tình hình sử dụng các hình thức "li" tù̀ li hợp tiếng Trung Quốc của sinh viên}

Từ li hợp tiếng Trung Quốc về cơ bản có 12 hình thức “li” sau (xem Bảng 1):

\section{Bảng 1}

Các hình thức "li" của từ li hợp tiếng Trung Quốc

\begin{tabular}{|c|c|}
\hline Mã số & Hình thức "li" \\
\hline $\mathrm{T} 1$ & hình vị "động” + trợ từ động thái 了/着/过 + hình vị "tân" \\
\hline $\mathrm{T} 2$ & hình vị "động" + trợ từ 的 + hình vị "tân" \\
\hline T3 & hình vị "động" + định ngữ mang tính thể từ + hình vị "tân" \\
\hline $\mathrm{T} 4$ & hình vị “động" + định ngữ mang tính vị từ + hình vị "tân" \\
\hline T5 & hình vị “động" + bổ ngữ chỉ hướng + hình vị "tân” \\
\hline T6 & hình vị "động” + bổ ngữ chỉ kết quả + hình vị "tân” \\
\hline $\mathrm{T} 7$ & hình vị "động” + bổ ngữ chỉ số lượng + hình vị "tân" \\
\hline $\mathrm{T} 8$ & hình vị "động" + bổ ngữ chỉ khả năng + hình vị "tân" \\
\hline T9 & hình vị "động” + bổ ngữ chỉ tình thái + hình vị "tân" \\
\hline $\mathrm{T} 10$ & hình vị "động” + bổ ngữ chỉ mức độ + hình vị "tân" \\
\hline $\mathrm{T} 11$ & hình vị "động” + hình vị "động" + hình vị "tân" \\
\hline $\mathrm{T} 12$ & hình vị "tân” + hình vị "động” \\
\hline
\end{tabular}


Nguồn: Tổng hợp của tác giả

Tình hình sử dụng các hình thức "li" này của sinh viên Việt Nam ở các giai đoạn sơ cấp, trung cấp, cao cấp như sau (xem Bảng 2):

\section{Bảng 2}

Tình hình sử dụng các hình thức "li" từ li hợp tiếng Trung Quốc của sinh viên Việt Nam qua các giai đoạn

\begin{tabular}{|c|c|c|c|c|c|c|c|c|c|}
\hline \multirow{2}{*}{ HTL } & \multicolumn{3}{|c|}{ So cấp } & \multicolumn{3}{c|}{ Trung cấp } & \multicolumn{3}{c|}{ Cao cấp } \\
\cline { 2 - 11 } & TSSD & TSSDCX & TLCX & TSSD & TSSDCX & TLCX & TSSD & TSSDCX & TLCX \\
\hline T1 & 24 & 17 & 70.8 & 26 & 23 & 88.5 & 18 & 15 & 83.3 \\
\hline T2 & 4 & 0 & 0.0 & 6 & 0 & 0.0 & 3 & 0 & 0.0 \\
\hline T3 & 28 & 19 & 67.9 & 46 & 34 & 73.9 & 14 & 13 & 92.9 \\
\hline T4 & 11 & 10 & 90.9 & 22 & 22 & 100.0 & 9 & 9 & 100.0 \\
\hline T5 & 8 & 3 & 37.5 & 6 & 4 & 66.7 & 4 & 1 & 25.0 \\
\hline T6 & 17 & 13 & 76.5 & 6 & 4 & 66.7 & 4 & 4 & 100.0 \\
\hline T7 & 19 & 14 & 73.7 & 42 & 33 & 78.6 & 21 & 20 & 95.2 \\
\hline T8 & 10 & 9 & 90.0 & 9 & 8 & 88.9 & 4 & 3 & 75.0 \\
\hline T9 & 6 & 0 & 0.0 & 3 & 0 & 0.0 & 2 & 0 & 0.0 \\
\hline T10 & 0 & 0 & 0.0 & 0 & 0 & 0.0 & 2 & 2 & 100.0 \\
\hline T11 & 15 & 13 & 86.7 & 12 & 10 & 83.3 & 8 & 8 & 100.0 \\
\hline T12 & 0 & 0 & 0.0 & 0 & 0 & 0.0 & 5 & 5 & 100.0 \\
\hline
\end{tabular}

Ghi chú: HTL là hình thức "li", TSSD là tần suất sử dụng, TSSDCX là tần suất sử dụng chính xác, TLCX là tỉ lệ chính xác. TLCX $=($ TSSDCX x 100)/ TSSD

Nguồn: Kết quả phân tích ngữ liệu của tác giả

\section{viên Việt Nam \\ 4.2. Xây dụng thứ tụ thụ đắc các hình thức "li” tù̀ li hộp tiếng Trung Quốc của sinh}

\subsubsection{Thư tụ tần suất sủ dụng chính xác}

Từ Bảng 2, chúng tôi có tần suất sử dụng chính xác của sinh viên Việt Nam ở cả ba giai đoạn (sơ cấp, trung cấp và cao cấp) và thứ tự từ cao xuống thấp của chúng như sau (xem Bảng 3 ):

\section{Bảng 3}

Tần suất sử dụng chính xác các hình thức “li” từ li hợp tiếng Trung Quốc của sinh viên Việt Nam

\begin{tabular}{|c|c|c|c|c|c|c|c|c|c|c|c|c|}
\hline & T1 & T2 & T3 & T4 & T5 & T6 & T7 & T8 & T9 & T10 & T11 & T12 \\
\hline TSSDCX & 55 & 0 & 66 & 41 & 8 & 21 & 67 & 20 & 0 & 2 & 31 & 5 \\
\hline Thứ tụ & 3 & 11 & 2 & 4 & 8 & 6 & 1 & 7 & 12 & 10 & 5 & 9 \\
\hline
\end{tabular}

Ghi chú: TSSDCX là tần suất sử dụng chính xác

Nguồn: Kết quả phân tích ngữ liệu của tác giả

Bảng 3 cho thấy, thứ tự từ cao xuống thấp của các hình thức "li" từ li hợp theo tần suất sử dụng chính xác như sau: 


\section{(1) $\mathrm{T} 7>\mathrm{T} 3>\mathrm{T} 1>\mathrm{T} 4>\mathrm{T} 11>\mathrm{T} 6>\mathrm{T} 8>\mathrm{T} 5>\mathrm{T} 12>\mathrm{T} 10>\mathrm{T} 2>\mathrm{T} 9$}

\subsubsection{Thư tư theo ma trận Guttman}

Chúng tôi lấy giá trị chuẩn là 0.6 (tức $60 \%$ ), chuyển hoá tỉ lệ sử dụng chính xác của hình thức "li" ở một giai đoạn ngôn ngữ về 0 và 01 . Khi tỉ lệ chính xác của hình thức "li" ở giai đoạn ngôn ngữ nào đó có giá trị nhỏ hơn giá trị chuẩn (tức $<0.6$ ), thì chuyển hoá thành 0 , nhận định rằng sinh viên ở giai đoạn ngôn ngữ đang xét chưa thụ đắc hình thức "li" này. Khi tỉ lệ chính xác của hình thức "li” ở giai đoạn ngôn ngữ nào đó có giá trị lớn hơn hoặc bằng giá trị chuẩn (tức $\geq$ 0.6 ), thì chuyển hoá thành 1 , nhận định rằng sinh viên ở giai đoạn ngôn ngữ đang xét đã thụ đắc hình thức "li" này. Với những hình thức "li" chưa xuất hiện ở một giai đoạn ngôn ngữ nào đó, tức không thể tính toán tỉ lệ chính xác, thì xem như sinh viên chưa thụ đắc và chuyển hoá thành 0 .

Sau khi chuyển các số liệu về tỉ lệ chính xác trong Bảng 2 về hai giá trị 0 và 01 , chúng tôi có được Bảng 4.

\section{Bảng 4}

Số liệu tỉ lệ chính xác ở các giai đoạn sau khi chuyển hoá

\begin{tabular}{|c|c|c|c|c|c|c|c|c|c|c|c|c|}
\hline & T1 & T2 & T3 & T4 & T5 & T6 & T7 & T8 & T9 & T10 & T11 & T12 \\
\hline Sơ cấp & 1 & 0 & 1 & 1 & 0 & 1 & 1 & 1 & 0 & 0 & 1 & 0 \\
\hline Trung cấp & 1 & 0 & 1 & 1 & 1 & 1 & 1 & 1 & 0 & 0 & 1 & 0 \\
\hline Cao cấp & 1 & 0 & 1 & 1 & 0 & 1 & 1 & 1 & 0 & 1 & 1 & 1 \\
\hline
\end{tabular}

Nguồn: Kết quả xử lí số liệu của tác giả

Từ Bảng 4, chúng tôi tiến hành sắp xếp theo ma trận Guttman và có được ma trận như Bảng 5.

\section{Bảng 5}

Ma trận Guttman các hình thức "li" của từ li hợp

\begin{tabular}{|c|c|c|c|c|c|c|c|c|c|c|c|c|c|}
\hline \multicolumn{8}{|c|}{ Dế } & \multicolumn{6}{|c|}{ Khó } \\
\hline & T4 & T11 & T8 & T7 & T1 & T6 & T3 & T5 & T12 & T10 & $\mathbf{T} 2$ & T9 & Tổng \\
\hline Cao cấp & 1 & 1 & 1 & 1 & 1 & 1 & 1 & 0 & 1 & 1 & 0 & 0 & \\
\hline Trung cấp & 1 & 1 & 1 & 1 & 1 & 1 & 1 & 1 & 0 & 0 & 0 & 0 & \\
\hline So cấp & 1 & 1 & 1 & 1 & 1 & 1 & 1 & 0 & 0 & 0 & 0 & 0 & \\
\hline Đúng & 3 & 3 & 3 & 3 & 3 & 3 & 3 & 1 & 1 & 1 & 0 & 0 & 24 \\
\hline Sai & 0 & 0 & 0 & 0 & 0 & 0 & 0 & 1 & 0 & 0 & 0 & 0 & 1 \\
\hline
\end{tabular}

Nguồn: Kết quả xử lí số liệu của tác giả

Bảng 5 có các chỉ tiêu hệ số tương quan như sau:

Hệ số tái lập (Crep):

Crep = 1 - Số lượng sai/(số lượng hình thức "li" x số lượng cấp độ)

$$
=1-1 /(12 \times 3)=0.972
$$

Hệ số tái lập biên tối thiểu (MMrep):

MMrep = Số lượng đúng/ (số lượng hình thức "li" x số lượng cấp độ) 


$$
=24 /(12 \times 3)=0.667
$$

Phần trăm cải thiện hệ số tái lập (\% IR):

$$
\begin{aligned}
\% \text { IR } & =\text { Crep }- \text { MMrep } \\
& =0.972-0.667=0.306
\end{aligned}
$$

Hệ số khả năng phân cấp (Cscal):

$$
\begin{aligned}
\text { Cscal } & =\% \text { IR/ }(1-\text { MMrep }) \\
& =0.306 /(1-0.667)=0.917
\end{aligned}
$$

Hệ số tái lập Crep $=0.972(>0.9)$. Điều này cho thấy, việc dựa vào ma trận để dự đoán những biểu hiện ngôn ngữ của sinh viên Việt Nam có tỉ lệ chính xác đến $97.2 \%$. Hệ số khả năng phân cấp Cscal là 0.917 , lớn hơn ngưỡng hiệu quả 0.6 . Điều này cho thấy ma trận Guttman các hình thức "li" của từ li hợp có hiệu quả, có thể phân cấp, cấp độ thật sự của ma trận Guttman (cấp độ khó và cấp độ năng lực) có giá trị tham khảo về mặt dự đoán.

Bảng 5 còn cho thấy, thứ tự các hình thức "li" của từ li hợp theo ma trận Guttman như sau:

$$
\mathrm{T} 4>\mathrm{T} 11>\mathrm{T} 8>\mathrm{T} 7>\mathrm{T} 1>\mathrm{T} 6>\mathrm{T} 3>\mathrm{T} 5>\mathrm{T} 12>\mathrm{T} 10>\mathrm{T} 2>\mathrm{T} 9
$$

\subsubsection{Thứ tụ thụ đắc các hình thức "li” tù̀ li hợp tiếng Trung Quốc}

Từ 4.2.1 và 4.2.2, chúng tôi có được hai dãy thứ tự sau:

$$
\begin{aligned}
& \mathrm{T} 7>\mathrm{T} 3>\mathrm{T} 1>\mathrm{T} 4>\mathrm{T} 11>\mathrm{T} 6>\mathrm{T} 8>\mathrm{T} 5>\mathrm{T} 12>\mathrm{T} 10>\mathrm{T} 2>\mathrm{T} 9 \\
& \mathrm{~T} 4>\mathrm{T} 11>\mathrm{T} 8>\mathrm{T} 7>\mathrm{T} 1>\mathrm{T} 6>\mathrm{T} 3>\mathrm{T} 5>\mathrm{T} 12>\mathrm{T} 10>\mathrm{T} 2>\mathrm{T} 9
\end{aligned}
$$

Quan sát hai dãy thứ tự này, chúng tôi nhận thấy:

Thứ nhất, các hình thức "li” T4, T11, T8, T7, T1, T6, T3 luôn đứng trong bảy vị trí đầu của hai dãy thứ tự. Có thể nói, đây là bảy hình thức "li" mà sinh viên dễ thụ đắc nhất. Sinh viên Việt Nam ngay từ giai đoạn sơ cấp đã có thể thụ đắc các hình thức "li" này.

Thứ hai, các hình thức "li" T2, T9 luôn đứng trong hai vị trí cuối của hai dãy thứ tự. Ma trận Guttman cho thấy, hai hình thức này sinh viên đều có tỉ lệ chính xác ở các giai đoạn thấp hơn giá trị chuẩn. Có thể nói, đây là hai hình thức "li" khó thụ đắc nhất. Sinh viên Việt Nam đến giai đoạn cao cấp vẫn chưa thể thụ đắc hai hình thức "li" này.

\begin{tabular}{|c|c|c|c|c|c|c|c|c|c|c|c|c|}
\hline $\mathrm{C \boxplus}$ & \multicolumn{7}{|c|}{ I } & \multicolumn{3}{|c|}{ II } & \multicolumn{2}{|c|}{ III } \\
\hline TT & 1 & 2 & 3 & 4 & 5 & 6 & 7 & 8 & 9 & 10 & 11 & 12 \\
\hline HTL & $\mathrm{T} 4$ & $\mathrm{~T} 11$ & T8 & $\mathrm{T} 7$ & $\mathrm{~T} 1$ & T6 & $\mathrm{T} 3$ & T5 & T12 & $\mathrm{T} 10$ & $\mathrm{~T} 2$ & T9 \\
\hline
\end{tabular}

Thứ ba, các hình thức "li" T5, T12, T10 luôn đứng ở ba vị trí giữa của hai dãy thứ tự. Ma trận Guttman cho thấy, hình thức T5 sinh viên có tỉ lệ chính xác cao hơn giá trị chuẩn ở giai đoạn trung cấp, hình thức T12 và T10 sinh viên có tỉ lệ chính xác cao hơn giá trị chuẩn ở giai đoạn cao cấp. Có thể nói, đây là ba hình thức "li" tương đối khó thụ đắc. Sinh viên Việt Nam đến giai đoạn trung, cao cấp mới có thể thụ đắc ba hình thức "li" này.

\section{Bảng 6}

Thứ tự thụ đắc các hình thức "li” từ li hợp của sinh viên Việt Nam

Ghi chú: CĐ là cấp độ, TT là thứ tự, HTL là hình thức "li"

Nguồn: Kết quả xử lí số liệu của tác giả

Trên cơ sở các phân tích trên đây, chúng tôi có được thứ tự thụ đắc các hình thức "li" từ 
li hợp tiếng Trung Quốc của sinh viên Việt Nam như Bảng 6. Trong đó, các hình thức "li" ở cấp độ I là các hình thức "li" dễ thụ đắc nhất, các hình thức "li" ở cấp độ II là các hình thức "li" tương đối khó thụ đắc, các hình thức "li" ở cấp độ III là các hình thức "li” khó thụ đắc nhất.

\subsection{So sánh với nghiên cứu trước đây}

Như đã đề cập ở phần mở đầu, tiêu chí xây dựng thứ tự thụ đắc của Ngo (2007) đã vi phạm nguyên lí thống kê. Vì vậy, chúng tôi cho rằng không cần thiết phải so sánh kết quả nghiên cứu của chúng tôi với nghiên cứu này. Trong phần này, chúng tôi tiến hành so sánh kết quả nghiên cứu của mình với kết quả nghiên cứu của $\mathrm{Ma} \mathrm{(2008).}$

Nghiên cứu của Ma (2008) hướng đến sinh viên quốc tế đang học tập tại Trung Quốc. Nghiên cứu này xuất phát từ góc độ thống kê học, thu thập ngữ liệu người học thông qua phiếu điều tra, đề cập đến 10 hình thức "li" của từ li hợp là "hình vị "động' + trợ từ + hình vị 'tân"”, "hình vị 'động' + bổ ngữ chỉ số lượng + hình vị 'tân", "hình vị 'động' + bổ ngữ chỉ khả năng + hình vị 'tân’”, "hình vị ‘động' + bổ ngữ chỉ hướng + hình vị 'tân’”, "hình vị 'động' + bổ ngữ chỉ kết quả + hình vị 'tân’”, "hình vị 'động' + hình vị 'động' + hình vị 'tân'”, "hình vị 'tân' + hình vị 'động"”, "hình vị ‘động' + tính từ + hình vị 'tân”, "hình vị 'động' + đại từ + hình vị 'tân’”, “hình vị 'động' + 什公 + hình vị 'tân’”.

Có thể thấy, trong 10 hình thức "li" mà Ma (2008) tiến hành khảo sát, chỉ có 06 hình thức "li" giống với cách phân loại của chúng tôi. Đó là các hình thức "li”: (T5) hình vị "động" + bổ ngữ chỉ hướng + hình vị "tân", (T6) hình vị "động” + bổ ngữ chỉ kết quả + hình vị "tân”, (T7) hình vị "động" + bổ ngữ chỉ số lượng + hình vị "tân", (T8) hình vị "động" + bổ ngữ chỉ khả năng + hình vị "tân", (T11) hình vị "động" + hình vị "động" + hình vị "tân", và (T12) hình vị "tân" + hình vị "động". Để tiện cho việc so sánh, thứ tự của các hình thức "li" này trong nghiên cứu của chúng tôi và nghiên cứu của $\mathrm{Ma}(2008)$ được sắp xếp lại từ 01 đến 06 (xem Bảng 7).

\section{Bảng 7}

So sánh kết quả giữa các nghiên cứu

\begin{tabular}{|c|c|c|c|c|c|c|}
\hline Thứ tự & $\mathbf{1}$ & $\mathbf{2}$ & $\mathbf{3}$ & $\mathbf{4}$ & $\mathbf{5}$ & $\mathbf{6}$ \\
\hline Nghiên cứu này & $\mathrm{T} 11$ & $\mathrm{~T} 8$ & $\mathrm{~T} 7$ & $\mathrm{~T} 6$ & $\mathrm{~T} 5$ & $\mathrm{~T} 12$ \\
\hline Ma (2008) & $\mathrm{T} 11$ & $\mathrm{~T} 7$ & $\mathrm{~T} 8$ & $\mathrm{~T} 5$ & $\mathrm{~T} 6$ & $\mathrm{~T} 12$ \\
\hline
\end{tabular}

Nguồn: Tổng hợp của tác giả

Bảng 7 cho thấy, kết quả nghiên cứu của chúng tôi và kết quả nghiên cứu của $\mathrm{Ma}$ (2008) giống nhau về thứ tự của $\mathrm{T} 11$ và $\mathrm{T} 12$, khác nhau về thứ tự giữa $\mathrm{T} 7$ và $\mathrm{T} 8$, giữa $\mathrm{T} 6$ và $\mathrm{T} 5$. Điều này phần nào cho thấy, thứ tự thụ đắc hình thức "li" từ li hợp của sinh viên Việt Nam tương tự với sinh viên quốc tế tại Trung Quốc. Mặt khác, kết quả này còn phản ánh việc sắp xếp thứ tự thụ đắc theo tiêu chí tần suất sử dụng chính xác và ma trận Guttman có độ tin cậy nhất định.

\section{Kết luận}

Với hai tiêu chí thứ tự tần suất sử dụng chính xác và thứ tự theo ma trận Guttman, trên cơ sở kết quả khảo sát Kho ngũ liệu ngôn ngũu trung gian tiếng Trung Quốc của sinh viên Việt Nam, chúng tôi có được thứ tự thụ đắc các hình thức "li" từ li hợp tiếng Trung Quốc của sinh viên Việt Nam như sau: (T4) hình vị "động" + định ngữ mang tính vị từ + hình vị "tân" > (T11) hình vị "động" + hình vị "động" + hình vị "tân" > (T8) hình vị "động" + bổ ngữ chỉ khả năng + hình vị "tân" > (T7) hình vị "động" + bổ ngữ chỉ số lượng + hình vị "tân" > (T1) hình vị "động" + trợ từ động thái 了/着/过 + hình vị “tân” > (T6) hình vị "động" + bổ ngữ chỉ kết quả + hình vị "tân" > 
(T3) hình vị "động” + định ngữ mang tính thể từ + hình vị "tân” > (T5) hình vị “động” + bổ ngữ chỉ hướng + hình vị "tân" > (T12) hình vị "tân" + hình vị "động" > (T10) hình vị "động" + bổ ngữ chỉ mức độ + hình vị "tân" > (T2) hình vị "động" + trợ từn + hù̀nh vị "tân" > (T9) hình vị “động” + bổ ngữ chỉ tình thái + hình vị "tân”. Trong đó, T4, T11, T8, T7, T1, T6, T3 là các hình thức "li" dễ thụ đắc, sinh viên Việt Nam đã có thể thụ đắc ngay từ giai đoạn sơ cấp; T5, T12, T10 là các hình thức "li" tương đối khó thụ đắc, sinh viên Việt Nam sang giai đoạn trung, cao cấp mới có thể thụ đắc; T2, T9 là các hình thức "li" khó thụ đắc, sinh viên Việt Nam đến giai đoạn cao cấp vẫn chưa thể thụ đắc.

Kết quả nghiên cứu này có giá trị tham khảo nhất định trong việc biên soạn giáo trình và đề thi tiếng Trung Quốc cho sinh viên Việt Nam. Khi biên soạn giáo trình tiếng Trung Quốc dành cho sinh viên Việt Nam, người biên soạn có thể căn cứ vào kết quả nghiên cứu này, sắp xếp trình tự xuất hiện các điểm ngữ pháp có liên quan đến hình thức "li" của từ li hợp, góp phần giúp sinh viên "tăng tốc" thụ đắc các điểm ngữ pháp này. Khi biên soạn các câu hỏi trong đề thi đánh giá năng lực tiếng Trung Quốc dành cho sinh viên Việt Nam, người biên soạn cũng có thể căn cứ vào kết quả nghiên cứu này, xác định độ khó của các câu hỏi về hình thức "li" của từ li hợp, lựa chọn câu hỏi phù hợp với trình độ tiếng Trung Quốc của sinh viên ở từng giai đoạn.

\section{Tài liệu tham khảo}

Huang, Z. R., \& Xiao, X. Q. (2012). Jiyu zhongjieyu yuliaoku de Hanguo xuesheng "ba” zi ju xide yanjiu [Acquisition of "Ba" construction of Korean students based on interlanguage corpus]. Chinese Language Learning, (1), 71-79.

Krashen, S. D. (1985). The input hypothesis: Issues and implications. New York, NY: Longman.

Luu, V. H. (2016). Yuenan xuesheng Hanyu quxiang buyu ju xide shunxu kaocha [A Study on the order of Vietnamese learner acquiring directional complements]. Shijie Huawen Jiaoxue, 2, 72-86.

Luu, V. H. (2020). Hanyue quxiang buyu duibi xide yanjiu [A comparative study of Chinese and Vietnamese directional complements and Vietnamese students' acquisition]. Beijing, China: Social Sciences Academic Press.

Luu, V. H. (2021). Khảo sát lỗi sử dụng từ li hợp tiếng Trung Quốc của sinh viên Việt Nam [An analysis of errors in Chinese separable words by Vietnamese]. Language and Life, (2), 6367.

Ma, P. (2008). Liuxuesheng dongbinshi liheci xide yanjiu - yi tongjixue wei shijiao [An analysis onthe acquisition of separable verbs]. Chinese Language Learning, (5), 108-112.

Ngo, H. T. L. (2007). Yuenan xuesheng Hanyu dongbinshi liheci xide yanjiu yu jiaoxue duice [Study on the acquisition of Chinese verb-object separate words and teaching strategies for Vietnamese students] (Master's thesis). Beijing Language and Culture University, Beijing, China.

Nguyen, H. L. U. (2019). Dui Yue Hanyu jiaoxue zhong de liheci yanjiu [Research on separable words in Vietnamese - Chinese teaching] (Doctoral dissertation). Central China Normal University, Wuhan, China.

Nguyen, H. T. T. (2017). Yuenan xuesheng dongbinshi liheci dai buyu xide yanjiu [Analysis of the acquisition of the verb-object clutch verb with complement of Vietnamese students] (Master's thesis). Shanghai International Studies University, Shanghai, China. 
Shi, J. W. (2012). Di'er yuyan xuexizhe Hanyu tezhi wenju xide de fazhan guocheng yanjiu [A study on the development process of second language learners' acquisition of Chinese specific questions]. Chinese L2 Theory and Practice: Proceedings of CLEF2011, 84-94. Santa Clara, CA: CLERC Publishing.

Truong, N. V. (2017). Yuenan xuesheng xide Hanyu liheci de pianwu kaocha yu diaocha duice [An investigation and investigation of the errors made by Vietnamese students in the acquisition of Chinese separate words] (Master's thesis). Liaoning Normal University, Dalian, China.

Vuong, T. Q. (2008). Yuenan xuesheng liheci shiyong pianwu fenxi [Phenomenology analyst be born Viet Nam is misapplied “Liheci-离合词”] (Master's thesis). Guangxi Normal University, Guilin, China.

Xiao, X. Q., \& Zhou, W. H. (2009). Waiguo xuesheng Hanyu quxiang buyu ju xide yanjiu [Acquisition study of the directional complement]. Chinese Language Learning, (1), 70-81.

Yang, D. F. (2003). Yingyu muyu xuexizhe quxiang buyu de xide shunxu [Sequence of acquiring the directional complement by English-speaking learners of Chinese]. Chinese Teaching in the World, (2), 52-65.

Zhang, H. C., \& Xu, J. P. (2009). Xiandai xinli yu jiaoyu tongjixue [Modern psychology and educational statistics]. Beijing, China: Beijing Normal University Publishing House. 


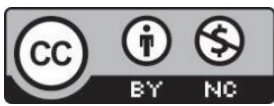

Creative Commons Attribution-NonCommercial 4.0 International License. 